\title{
An Input-Constrained NARMAX Model-Based Adaptive Tracker with Fault Tolerance for Unknown Systems with an Input-Output Direct Feed-Through Term
}

\author{
${ }^{1}$ Jason Sheng-Hong Tsai, ${ }^{1}$ Wen-Teng Hsu, ${ }^{1}$ Ya-Chiou Hsu, ${ }^{2}$ Shu-Mei Guo, ${ }^{3}$ Tzong-Jiy Tsai, ${ }^{4}$ Jose I. Canelon, ${ }^{5}$ Leang-San \\ Shieh
}

${ }^{1}$ National Cheng Kung University Department of Electrical Engineering, Tainan 701, Taiwan, R.0.C., 011-886-6-2757575-62360

${ }^{2}$ National Cheng Kung University Department of Computer Science and Information Engineering, Tainan 701, Taiwan, R.O.C., 011-886-6-2757575-62525

${ }^{3}$ Tung-Fang Design Institute Department of Game and Toy Design, Kaohsiung 82941, Taiwan, R.O.C.

${ }^{4}$ Universidad del Zulia Electrical Engineering School, Maracaibo 4005, Venezuela

${ }^{5}$ University of Houston Department of Electrical and Computer Engineering, Houston, Texas 77204-4005, U.S.A.

This work was supported by the Ministry of Science and Technology of Republic of China under Contracts MOST 105-2221-E-006-102-MY3 and MOST 106-

2221-E-006-242.

Correspondence Author: Jason Sheng-Hong Tsai, National Cheng Kung University Department of Electrical Engineering, Tainan 701, Taiwan, R.O.C., 011-886-6-2757575-62360

Received date: 12 January 2018, Accepted date: 10 March 2018, Online date: 20 March 2018

Copyright: (C) 2018 Jason Sheng-Hong Tsai et al. This is an open-access article distributed under the terms of the Creative Commons Attribution License, which permits unrestricted use, distribution, and reproduction in any medium, provided the original author and source are credited.

\begin{abstract}
A modified NARMAX (nonlinear autoregressive moving average with exogenous inputs) model-based state-space self-tuner with input-constrained fault tolerance is proposed in this paper for unknown nonlinear stochastic hybrid systems with an input-output direct transmission term. First, the on-line system identification process is enhanced by using the off-line observer/Kalman filter identification method to obtain a good initial guess of the modified NARMAX model. Then, based on such system identification, a corresponding adaptive digital control scheme is presented for an unknown continuous-time nonlinear proper system with system and measurement noises and inaccessible system states. Besides, an effective state space self-turner with a fault tolerant scheme is presented for the unknown multivariable stochastic system. Further, a quantitative criterion is used to develop a weighting matrix resetting technique by adjusting and resetting the covariance matrices of the parameters estimated by the Kalman filter estimation algorithm, to achieve parameter estimation for the recovery of the faulty system. Finally, the proposed new anti-windup scheme according to these models set up decentralized trajectory trackers for unknown interconnected large-scale systems with input constraints and state delays. An illustrative example is given to demonstrate the effectiveness of the proposed method.
\end{abstract}

Key words: Self-Tuning Control, NARMAX Model, Fault Tolerant Control, OKID, RELS, Input Constraint

\section{INTRODUCTION}

The state-space self-tuning control methods [1-2] have shown to be effective in designing advanced adaptive controllers for linear multivariable stochastic systems [3-6]. In those approaches [1-2], the standard Kalman state-estimation algorithm [7] has been embedded into an on-line parameter estimation algorithm. Now, if the dynamics of the system is significantly nonlinear, a nonlinear model may in general be required for system analysis and control design. This immediately raises the question of what class of models to use. Polynomial expansions are used extensively in nonlinear system analysis, when the system has no input-to-output direct feed-through term. On the other hand, the traditional NARMAX model, which was first introduced and rigorously derived by Leontaritis and Billings [8], provides a unified representation for a wide class of nonlinear stochastic systems [9]. The NARMAX model is not restricted to polynomial systems and can be expanded as a rational model [10]. The advantage of the rational model is its efficiency in describing severe nonlinear characteristics with few parameters. These results can be related to the models introduced by Sontag [11]. When they are extended to the case of unknown stochastic systems, they provide a class of rational models [12] which can be used as the basis of parameter estimation algorithms.

Now, over the past decades there has been a growing interest in the study of singular systems due to their extensive use in large-scale systems, circuits, power systems, economics, control theory, robots, and other areas. The tracker and fault tolerant control for linear singular systems is given in Wang et al [13]. A singular system can be converted into an equivalent regular system which may have an input-to-output direct transmission term. Indeed, a singular system without the impulse mode is just a special class of regular systems with the direct transmission term.

Practical systems usually exhibit hard physical constraints, especially on the actuator. Hence, designing high performance feedback controllers for these systems might easily induce actuator saturation [14]. The inability to resolve the input saturation problem leads to the undesirable system behavior (e.g. excessive overshoot, oscillation and instability) known as wind-up. An anti-windup control scheme is a popular approach to deal with this issue. Some works [15-16] propose systematic methodologies for compensation design based on optimization theory. Some others [17-18] present a synthesis method combining the nominal controller with the anti-windup compensator to guarantee the stability and performance of closed-loop systems [17-18]. In addition, model predictive control has been applied to resolve constrained linear and quadratic optimization problems [19-20]. To the author's knowledge, the NARMAX model based state-space optimal tracker with fault tolerance for a nonlinear sampled-data system containing an input-to-output direct transmission term and input constraints, has not yet been proposed in the literature.

This paper is organized as follows. Problem description is given in Sec. 2. Section 3 presents a new optimal anti-windup tracker for a system with an inputto-output direct transmission term. An illustrative example is shown in Sec. 4 and conclusion is given in Sec. 5. 
Citation: Jason Sheng-Hong Tsai, Wen-Teng Hsu, Ya-Chiou Hsu, Shu-Mei Guo, Tzong-Jiy Tsai, Jose I. Canelon, Leang-San Shieh, 2018. An Input-Constrained NARMAX Model-Based Adaptive Tracker with Fault Tolerance for Unknown Systems with an Input-Output Direct Feed-Through Term. Advances in Natural and Applied Sciences. 12(3): 8-20.

Problem Description:

Consider the continuous-time nonlinear stochastic systems (see Fig. 1):

Sys 1: $\dot{x}_{1}(t)=f_{1}\left(x_{1}(t)\right)+g_{1}\left(x_{1}(t)\right) u_{1}(t)+\xi_{1}\left(y_{2}(t)\right)+w_{1}^{\prime}(t)$,

$$
y_{1}(t)=h_{1}\left(x_{1}(t)\right)+d_{1}\left(x_{1}(t)\right) u_{1}(t)+v_{1}^{\prime}(t)
$$

Sys $2: \dot{x}_{2}(t)=f_{2}\left(x_{2}(t)\right)+g_{2}\left(x_{2}(t)\right) u_{2}(t)+\xi_{2}\left(y_{1}(t)\right)+w_{2}^{\prime}(t)$,

$$
y_{2}(t)=h_{2}\left(x_{2}(t)\right)+d_{2}\left(x_{2}(t)\right) u_{2}(t)+v_{2}^{\prime}(t) \text {, }
$$

where (1a)-(1b) are assumed unknown, $f_{i}: \Re^{n_{i}} \rightarrow \mathfrak{R}^{n_{i}}, g_{i}: \mathfrak{R}^{n_{i}} \rightarrow \mathfrak{R}^{n_{i} \times m_{i}}, h_{i}: \mathfrak{R}^{n_{i}} \rightarrow \mathfrak{R}^{p_{i}}, \quad d_{i}: \Re^{n_{i}} \rightarrow \mathfrak{R}^{n_{i} \times m_{i}}, i=1,2, u_{i}(k) \in \mathfrak{R}^{m_{i}}$ is the control input, $x_{i}(k) \in \mathfrak{R}^{n_{i}}$ is the state vector, $y_{i}(t) \in \mathfrak{R}^{p_{i}}$ is the measurable output vector, $w_{i}^{\prime}(t)$ and $v_{i}^{\prime}(t)$ are uncorrelated white noise processes. If an input-output feedthrough term is presented, a causality issue arises since the current control input $u\left(k T_{S}\right)$ is required for on-line system identification before it is determined. To overcome this problem, a modified NARMA model-based scheme is proposed in our previous work [21] for the identification of the unknown nonlinear system with the input-output feed-through term.

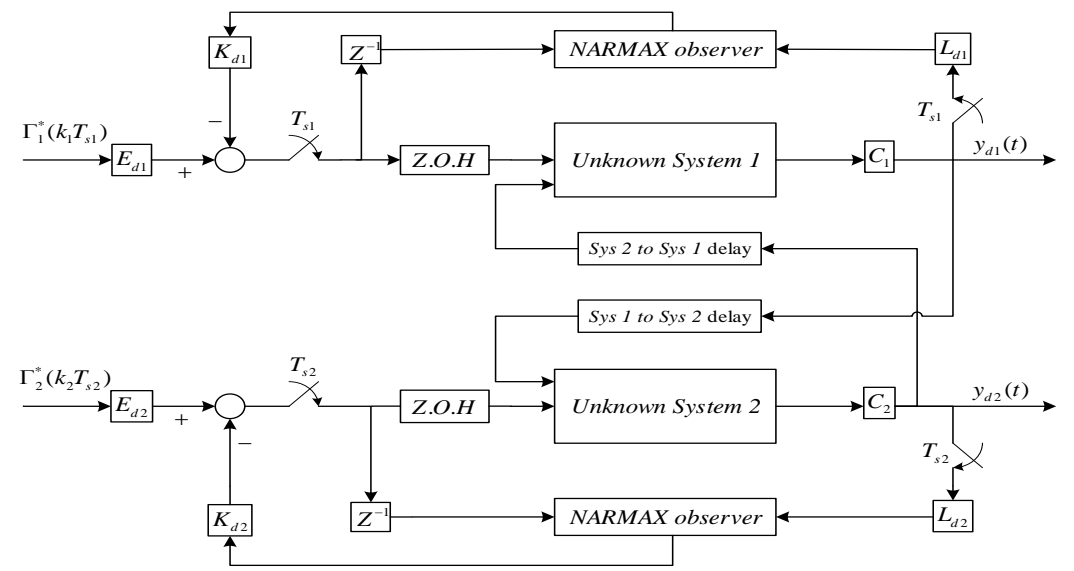

Fig. 1: Decentralized observer-based digital-redesign trackers for unknown sampled-data interconnected large-scale system (for $N=2$, where $T_{s 1}$ may not equal to $T_{s 2}$ ) with state and/or output delay terms.

Under this framework, the parameters and state of the unknown model are estimated from current and previous measurements of the control input $\left(u_{d}^{*}\left(k T_{s}\right), u_{d}\left((k-1) T_{s}\right), u_{d}\left((k-2) T_{s}\right), \cdots\right)$ and the system output $\left(y\left(k T_{s}\right), y\left((k-1) T_{s}\right), y\left((k-2) T_{s}\right), \cdots\right)$, where $u_{d}^{*}\left(k T_{s}\right)$ is to be estimated based on a predictionbased approach similar to one depicted in Fig. 2 (Tsai et al. 2016) for the linear case.

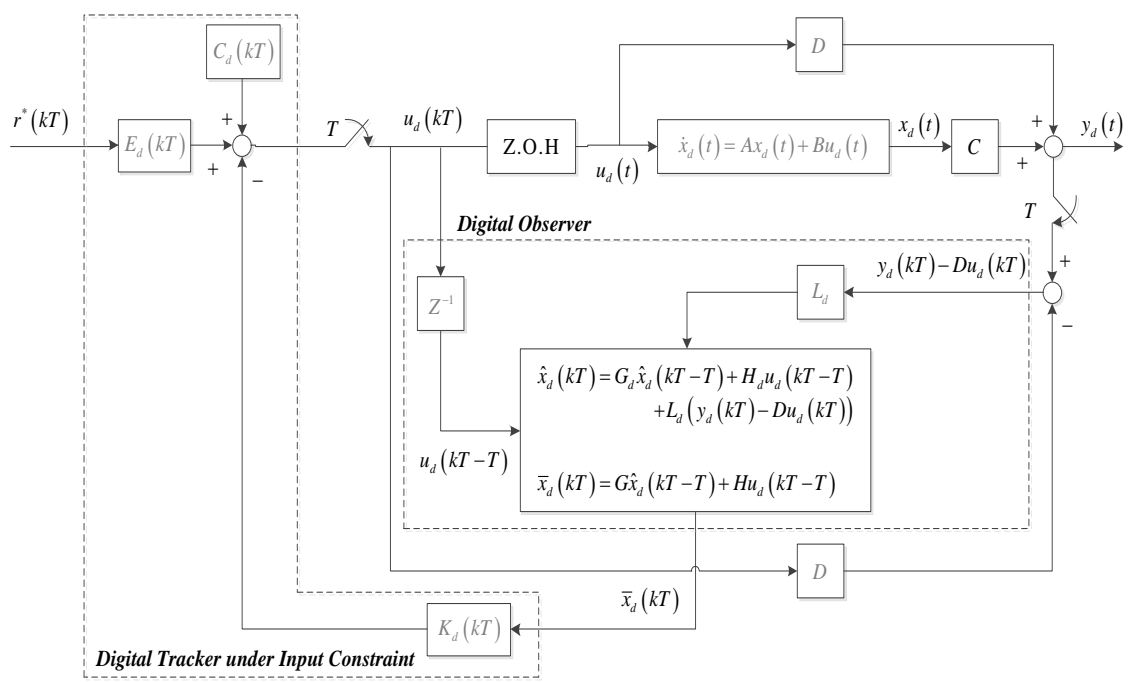

Fig. 2: Prediction-based digital observer and tracker for proper systems.

Considering the complexity of applying the optimal linearization method to linearize the NARMAX model as a linear ARMAX model at the operating state without any approximation, this paper uses the NARMAX model in polynomial expansion form, but not in rational expansion form. Based on the estimated modified NARMAX model, an appropriate controller can then be designed so that $y\left(k T_{s}\right) \rightarrow r\left(k T_{s}\right)$ for $k \geq 1$.

The polynomial expansion form of the modified NARMAX model for an m-input p-output system is given by 


$$
\begin{aligned}
\tilde{y}_{i}(k)=F_{i}\left[y_{1}(k-1), \cdots, y_{p}(k-1), \cdots, y_{1}\left(k-n_{y}\right), y_{2}\left(k-n_{y}\right), \cdots, y_{p}\left(k-n_{y}\right),\right. \\
u_{1}{ }^{*}(k), u_{2}{ }^{*}(k), \cdots, u_{m}{ }^{*}(k), u_{1}(k-1), u_{2}(k-1), \cdots, u_{m}(k-1), \cdots, \\
u_{1}\left(k-n_{u}\right), u_{2}\left(k-n_{u}\right), \cdots, u_{m}\left(k-n_{u}\right), \varepsilon_{1}(k-1), \cdots, \varepsilon_{p}(k-1), \cdots, \\
\left.\varepsilon_{1}\left(k-n_{e}\right), \varepsilon_{2}\left(k-n_{e}\right), \cdots, \varepsilon_{p}\left(k-n_{e}\right)\right],
\end{aligned}
$$

where $k(k=0,1, \cdots)$ is the time index, $\tilde{y}_{i}(k)$ is the estimated value of $y_{i}(k), u_{\alpha}(k)$ and $y_{\beta}(k)$ denote the $\alpha$-th input $(\alpha=1,2, \cdots, m)$ and the $\beta$-th output $(\beta=1,2, \cdots, p)$, respectively, and $\varepsilon_{\bullet}(k)$ is the residual. In addition, $n_{y}, n_{u}, n_{e}$ are the orders of $y, u, \varepsilon$, respectively, $\bar{n}$ is the number of linear/nonlinear variables $(y, u, \varepsilon)$ of the model, $\theta_{i j}(k)$ denotes the $j$-th coefficient for the $i$-th estimated output $\tilde{y}_{i}(k), \phi_{i j}(k)$ denotes the $j$-th linear/nonlinear variables $(y, u, \varepsilon)$ for the $i$-th estimated output $\tilde{y}_{i}(k), F_{i}(\bullet)$ are nonlinear polynomials for $i=1,2, \cdots, p, n_{\bullet}$ denotes the lags and $\phi_{\bullet}(k)$ can be any nonlinear function in $F_{i}(\bullet)$. A more specific expression of the modified NARMAX model for an $m$-input $p$-output system is newly presented in Appendix A.

Every estimated output $\tilde{y}_{i}(k)$ is calculated from each class of nonlinear $\phi_{i}(k)$ as

$\tilde{y}_{i}(k)=\theta_{i}^{T}(k) \phi_{i}(k), \quad i=1,2, \cdots, p$,

and the standard RELS algorithm given by

$$
\begin{aligned}
& \theta_{i}(k)=\theta_{i}(k-1)+\frac{S_{i}(k-1) \phi_{i}(k)}{\lambda(k)+\phi_{i}^{T}(k) S_{i}(k-1) \phi_{i}(k)} \varepsilon_{i}(k), \\
& S_{i}(k)=\frac{1}{\lambda(k)}\left(S_{i}(k-1)-\frac{S_{i}(k-1) \phi_{i}(k) \phi_{i}(k)^{T} S_{i}(k-1)}{\lambda(k)+\phi_{i}^{T}(k) S_{i}(k-1) \phi_{i}(k)}\right)
\end{aligned}
$$

is applied, where $\lambda(k)$ is a forgetting function to discount the old measurements, whose dynamics is described by the first-order difference equation, $\lambda(k)=\lambda_{0} \lambda(k-1)+\left(1-\lambda_{0}\right)$, with initial condition $0.9<\lambda(0)<1$, and updating factor $0<\lambda_{0}<1$. Also, $S_{i}(k) \in \mathfrak{R}^{\bar{n} \times \bar{n}}$ is the covariance matrix of the estimation errors on the parameters, with $S_{i}(0)=\alpha_{i} I_{\bar{n} \times \bar{n}}$, where $\alpha_{i}$ is the positive scalar, and the residual vector corresponding to each output is given by

$\varepsilon_{i}(k)=y_{i}(k)-\theta_{i}^{T}(k-1) \phi_{i}(k)$

Different classes of NARMAX models can be defined for different choices of $F_{i}(\cdot)$. A simplified complete control scheme for the complicate on-line faulttolerant control can be derived by choosing simple structures of dynamic nonlinear models, e.g. a self-tuning control scheme based on NARMAX models for nonlinear stochastic systems.

A New Anti-Windup Tracker For MIMO Systems Under Input Constraint With A Feed-Through Term:

The optimal anti-windup control scheme for strictly proper systems previously presented in Tsai et al. [10] has now been extended to the case of proper systems taking into consideration the input constraint issue.

The modified optimal control:

Consider the multi-input multi-output (MIMO) system

$\dot{x}_{T}(t)=A x_{T}(t)+B u_{T}(t)$,

$y_{T}(t)=C x_{T}(t)+D u_{T}(t)$,

where $x_{T}(t) \in \mathfrak{R}^{n}$ is the system state, $x_{T}(0)=x_{0} \in \mathfrak{R}^{n}$ is the initial state, $y_{T}(t) \in \mathfrak{R}^{p}$ is the system output and $u_{T}(t) \in \mathfrak{R}^{m}$ is the optimal control input which, according to the regular optimal control scheme, is designed to minimize the performance index

$J\left(t_{0}\right)=\frac{1}{2} \int_{t_{0}}^{T_{f}}\left\{\left[(C x(t)-\Gamma(t))+D u_{T}(t)\right]^{T} Q\left[(C x(t)-\Gamma(t))+D u_{T}(t)\right]+u_{T}^{T}(t) R u_{T}(t)\right\} d t$,

over the time interval $\left[t_{0}, t_{f}\right]$, where $Q$ are positive semi-definite matrices, $R$ is a positive definite matrix, $\Gamma(t) \in \mathfrak{R}^{p}$ is the reference input vector, $Q=q I_{p}>0$, and $R=r I_{m}>0$. Then, the corresponding regular optimal control input $u_{T}(t)$ is given by the state-feedback control law

$u_{T}(t)=-K_{c T} x_{T}(t)+E_{c T} \Gamma(t)$

where $K_{c T} \in \mathfrak{R}^{m \times n}$ is the analog feedback gain given by

$K_{c T}=\left(R+D^{T} Q D\right)^{-1}\left(B^{T} P_{T}+D^{T} Q C\right)$,

$E_{c T} \in \mathfrak{R}^{m \times p}$ is the forward gain calculated as

$E_{c T}=-\left(R+D^{T} Q D\right)^{-1}\left\{\left[\left(C-D K_{c T}\right)\left(A-B K_{c T}\right)^{-1} B\right]-D\right\}^{T} Q$, 
and $P_{T}$ is the symmetric positive definite solution of the Riccati equation

$$
\begin{gathered}
A^{T} P_{T}+P_{T} A+C^{T} Q C-\left(P_{T} B+C^{T} Q D\right)\left(R+D^{T} Q D\right)^{-1} . \\
\times\left(B^{T} P_{T}+D^{T} Q C\right)=0
\end{gathered}
$$

This regular optimal control scheme is depicted in Fig. 3.

The linear quadratic analog tracker (LQAT) can provide a good tracking performance thus making that the system output traces the desired reference as fast as possible, if the pair $(Q, R)$ is chosen to satisfy the high gain property $q \gg r$. However, if the given reference input $\Gamma(t)$ has significant variations at particular time instants, the tracker may easily produce large values of the input which might not fulfil the input constraint of some physical systems.

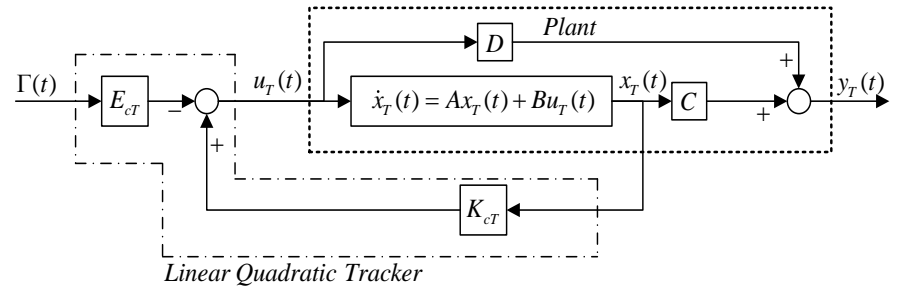

Fig. 3: Optimal LQAT of the regular analog model containing a feed-through term.

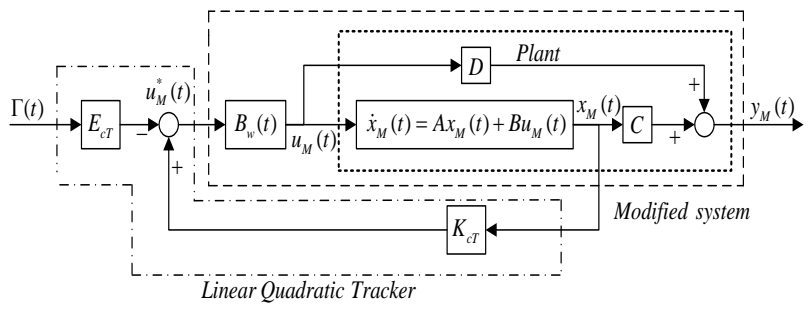

Fig. 4: Optimal LQAT of the modified analog model containing a feed-through term.

To overcome this disadvantage, the modified LQAT shown in Fig. 4 is proposed, which can effectively restrict the control input within the specified constraint interval, while exhibiting acceptable tracking performance. Let us assume that an input constraint vector $u_{\text {sat }}^{I}=\left[u_{\text {sat }, 1}^{I}, \cdots, u_{\text {sat }, i}^{I}, \cdots, u_{\text {sat }, m}^{I}\right] \in \mathfrak{R}^{m}$ is given, such that the control input $u_{T, i}(t) \in \mathfrak{R}$ should comply

$u_{T, i}(t)=\left\{\begin{array}{cl}\bar{u}_{i} & \text { if } u_{T, i}(t)>\bar{u}_{i}>0 \\ u_{T, i}(t) & \text { if } \underline{u}_{i} \leq u_{T, i}(t) \leq \bar{u}_{i} \\ \underline{u}_{i} & \text { if } u_{T, i}(t)<\underline{u}_{i}<0\end{array}\right.$,

where $u_{s a t, i}^{I}=\left[\bar{u}_{i}, \underline{u}_{i}\right]$, with $\bar{u}_{i}$ and $\underline{u}_{i}$ representing pre-specified upper and lower bounds, respectively of the corresponding $u_{T, i}(t)$. According to the proposed modified LQAT, if the amplitude of the control input $u_{T, i}(t)$ calculated based on the conventional LQAT exceeds the specified constraint $u_{\text {sat }, i}^{I}$ at a particular $t=t^{*}$, assign a forward weighted matrix

$B_{w}\left(t^{*}\right)=\frac{1}{\kappa\left(t^{*}\right)} I_{m}$

to $(5 a)-(5 b)$ to obtain the modified control system

$\dot{x}_{M}(t)=A x_{M}(t)+B B_{w}\left(t^{*}\right) u_{M}^{*}(t) \triangleq A x_{M}(t)+B^{*}\left(t^{*}\right) u_{M}^{*}(t)$,

$y_{M}(t)=C x_{M}(t)+D B_{w}\left(t^{*}\right) u_{M}^{*}(t) \triangleq C x_{M}(t)+D^{*}\left(t^{*}\right) u_{M}^{*}(t)$,

where $u_{M}^{*}(t) \in \mathfrak{R}^{m}$ is the modified control input determined, based on the system pair $\left\{A, B^{*}\left(t^{*}\right), C, D^{*}\left(t^{*}\right)\right\}$, to minimize the performance index, $B^{*}\left(t^{*}\right) \equiv B B_{w}\left(t^{*}\right)=\frac{1}{\kappa\left(t^{*}\right)} B, D^{*}\left(t^{*}\right) \equiv D B_{w}\left(t^{*}\right)=\frac{1}{\kappa\left(t^{*}\right)} D$, and $\kappa\left(t^{*}\right)=\max \left\{\kappa_{i}\left(t^{*}\right), i=1,2, \cdots, m\right\}$ with

$\kappa_{i}\left(t^{*}\right)=\left\{\begin{array}{cl}\frac{u_{T, i}\left(t^{*}\right)}{\bar{u}_{i}} & \text { if } u_{T, i}(t)>\bar{u}_{i}>0 \\ 1 & \text { if } \underline{u}_{i} \leq u_{T, i}(t) \leq \bar{u}_{i} \\ \frac{u_{T, i}\left(t^{*}\right)}{\underline{u}_{i}} & \text { if } u_{T, i}(t)<\underline{u}_{i}<0\end{array}\right.$

Notice that $u_{M}^{*}(t) \neq B_{w}^{-1}\left(t^{*}\right) u_{T}(t)$

Then, the corresponding optimal state-feedback control law is designed to minimize the performance index

$J\left(t_{0}\right)=\frac{1}{2} \int_{t_{0}}^{T_{f}}\left\{\left[(C x(t)-\Gamma(t))+D^{*}\left(t^{*}\right) u_{M}^{*}(t)\right]^{T} Q\left[(C x(t)-\Gamma(t))+D^{*}\left(t^{*}\right) u_{M}^{*}(t)\right]+u_{M}^{*}(t) R u_{M}^{*}(t)\right\} d t$, 
where $Q=q I_{p}>0, R=r I_{m}>0$, and $q \gg r$, which results in the modified controller

$u_{M}^{*}(t)=-K_{c M}\left(t^{*}\right) x_{M}(t)+E_{c M}\left(t^{*}\right) \Gamma(t)$,

where the analog feedback gain $K_{c M}\left(t^{*}\right) \in \mathfrak{R}^{m \times n}$ is given by

$K_{c M}\left(t^{*}\right)=\left(\kappa^{T}\left(t^{*}\right) R \kappa\left(t^{*}\right)+D^{T} Q D\right)^{-1}\left[B^{T} P_{M}\left(t^{*}\right)+D^{T} Q C\right]$

and the forward gain $E_{c M}\left(t^{*}\right) \in \mathfrak{R}^{m \times p}$ is calculated as

$$
\begin{aligned}
E_{c M}\left(t^{*}\right) & =-\left(\kappa^{T}\left(t^{*}\right) R \kappa\left(t^{*}\right)+D^{T} Q D\right)^{-1} \\
\times & \left.\times\left[\left(C-D K_{c M}\left(t^{*}\right)\right)\left(A-B K_{c M}\left(t^{*}\right)\right)^{-1} B\right]-D\right\}^{T} Q,
\end{aligned}
$$

where $P_{M}\left(t^{*}\right)$ is the re-solved symmetric positive definite solution of the Riccati equation

$$
\begin{aligned}
& A^{T} P_{M}\left(t^{*}\right)+P_{M}\left(t^{*}\right) A+C^{T} Q C-\left(P_{M}\left(t^{*}\right) B^{*}\left(t^{*}\right)+C^{T} Q D^{*}\left(t^{*}\right)\right) \times\left(R+D^{*^{T}}\left(t^{*}\right) Q D^{*}\left(t^{*}\right)\right)^{-1}\left(B^{*^{T}}\left(t^{*}\right) P_{M}\left(t^{*}\right)+D^{*^{T}}\left(t^{*}\right) Q C\right) \\
= & A^{T} P_{M}\left(t^{*}\right)+P_{M}\left(t^{*}\right) A+C^{T} Q C-\left(P_{M}\left(t^{*}\right) B+C^{T} Q D\right)\left(\kappa^{T}\left(t^{*}\right) R \kappa\left(t^{*}\right)+D^{T} Q D\right)^{-1}\left(B^{T} P_{M}\left(t^{*}\right)+D^{T} Q C\right)=0 .
\end{aligned}
$$

From the above formulation, the modified controller $u_{M}^{*}(t)$ exhibits good tracking performance, similar to the conventional high-gain-based tracker, and the real control input $u_{M}(t)=\frac{1}{\kappa\left(t^{*}\right)} u_{M}^{*}(t)$ fulfils the corresponding constraints.

Lemma 1: (Stability and optimality of the modified controller)

The proposed controller guarantees the stability of the closed-loop system (i.e. $\lambda\left(A-B^{*}\left(t^{*}\right) K_{c M}\left(t^{*}\right)\right)<0$, where $\lambda(\bullet)$ denotes the eigenvalues of $\left.(\cdot)\right)$, since the pair of matrices $\left(A, B^{*}\left(t^{*}\right)\right)$ is controllable if $(A, B)$ is controllable. In addition, from (11a)-(11b) the modified control system $\left\{A, B^{*}\left(t^{*}\right), C, D^{*}\left(t^{*}\right)\right\}$ with the weighting matrix pair $\{Q, R\}$ can be converted to the equivalent one $\{A, B, C, D\}$ with $\left\{Q, \hat{R}\left(t^{*}\right)\right\}=\left\{Q, \kappa^{T}\left(t^{*}\right) R \kappa\left(t^{*}\right)\right\}$. Deriving the optimality of the modified controller is trivial, since it is analogous to the derivation of the conventional optimal control theorem.

Characteristic of the modified control scheme:

According to the proposed controller design, a reasonable compression factor $\kappa\left(t^{*}\right)$ can be used to effectively restrict the actual input $u_{M}(t)$ within the prespecified constraint interval. To realize $\kappa\left(t^{*}\right)$, proofs of two features of the modified control scheme are required: (i) the upper bound $\bar{\kappa}$ of the compression factor $\kappa\left(t^{*}\right)$ and (ii) $u_{M}^{*}(t) \cong u_{T}(t)$.

Assumption 1:

For the modified controller presented in Sec. 3.1, since the weighting matrices $Q$ and $R$ are chosen to satisfy the high gain property, the features

$\left\|\left(P_{M}\left(t^{*}\right) B+C^{T} Q D\right)\left(\kappa^{T}\left(t^{*}\right) R \kappa\left(t^{*}\right)+D^{T} Q D\right)^{-1}\left(B^{T} P_{M}\left(t^{*}\right)+D^{T} Q C\right)\right\| \gg\left\|A^{T} P_{M}\left(t^{*}\right)\right\|$

and

$\left\|C^{T} Q C\right\| \gg\left\|P_{M}\left(t^{*}\right) A\right\|$

where $\|\cdot\|$ represents as the 2-norm of a matrix, can be deduced from Riccati equation.

Remark 1:

The conventional linear quadratic analog controller also exhibits the features of Assumption 1, i.e.

$\left\|\left(P_{T} B+C^{T} Q D\right)\left(R+D^{T} Q D\right)^{-1}\left(B^{T} P_{T}+D^{T} Q C\right)\right\| \gg\left\|A^{T} P_{T}\right\|$

and

$\left\|C^{T} Q C\right\| \gg\left\|P_{T} A\right\|$

while the weighting matrices $Q$ and $R$ are chosen to satisfy the high gain property. Based on Assumption 1 , while the weighting matrices are given as the diagonal matrices $Q=q I_{p}>0$ and $R=r I_{m}>0$, respectively, there exists a positive constant $\alpha>1$ such that the upper bound $\bar{\kappa}$ of the compression factor $\kappa\left(t^{*}\right)$ can be determined as

$\bar{\kappa}=\frac{1}{\alpha} \sqrt{\frac{\left\|B^{T} B\right\| \cdot\left\|C C^{T}\right\|}{\left\|A A^{T}\right\|} \cdot \frac{q}{r}}$

provided the property of the high-gain control, i.e. $P_{M}\left(t^{*}\right) \approx P_{T}$ (to appear in (28)), still holds.

The proof of the above observation is given as follows. Combining (16) and (17) results in the inequality $\left\|\left(P_{M}\left(t^{*}\right) B+C^{T} Q D\right)\left(\kappa^{T}\left(t^{*}\right) R \kappa\left(t^{*}\right)+D^{T} Q D\right)^{-1}\left(B^{T} P_{M}\left(t^{*}\right)+D^{T} Q C\right)\right\| \times\left\|C^{T} Q C\right\| \gg\left\|P_{M}\left(t^{*}\right) A A^{T} P_{M}\left(t^{*}\right)\right\|$, 
because $\left\|P_{M}\left(t^{*}\right) A\right\|\left\|A^{T} P_{M}\left(t^{*}\right)\right\| \geq\left\|P_{M}\left(t^{*}\right) A A^{T} P_{M}\left(t^{*}\right)\right\|$. Since the determination of the upper bound $\bar{\kappa}$ is independent of the $D$ term (see Remark 3$)$, equation (20) reduces to the $D$ term-free case as explained below. Due to the definition of norm, equation (20) implies that

$\left\|P_{M}\left(t^{*}\right) B\left(\kappa^{T}\left(t^{*}\right) R \kappa\left(t^{*}\right)\right)^{-1} B^{T} P_{M}\left(t^{*}\right)\right\| \cdot\left\|C^{T} Q C\right\|$

$\approx\left\|B\left(\kappa^{T}\left(t^{*}\right) R \kappa\left(t^{*}\right)\right)^{-1} B^{T}\right\| \cdot\left\|C^{T} Q C\right\| \gg\left\|A A^{T}\right\|$

to get

$\frac{q}{r \kappa^{2}\left(t^{*}\right)}\left\|B B^{T}\right\| \cdot\left\|C^{T} C\right\| \gg\left\|A A^{T}\right\|$

where $Q=q I_{p}>0$ and $R=r I_{m}>0$ are diagonal matrices. Finally, rearranging (22) gives

$\kappa\left(t^{*}\right) \ll \sqrt{\frac{\left\|B^{T} B\right\| \cdot\left\|C C^{T}\right\|}{\left\|A A^{T}\right\|} \cdot \frac{q}{r}}$.

With (23), there exists a positive constant $\alpha>1$ to define the upper bound $\bar{\kappa}$ of the compression factor $\kappa\left(t^{*}\right)$ as

$\bar{\kappa}=\frac{1}{\alpha} \sqrt{\frac{\left\|B^{T} B\right\| \cdot\left\|C C^{T}\right\|}{\left\|A A^{T}\right\|} \cdot \frac{q}{r}}$

provided the property of high-gain control, i.e. $P_{M}\left(t^{*}\right) \approx P_{T}$ (to appear in (28)), still holds.

More specifically, the above observation with proof can be summarized in

Theorem 1:

Given the analog system in terms of matrices $\{A, B, C, D\}$, let the weighting matrices be diagonal matrices $Q=q I_{P}>0, R=r I_{m}>0$, and $q \gg r$. There exists the lower bound $\left\{Q^{*}, R^{*}\right\}$ of weighting matrices, i.e. $Q^{*}=q^{*} I_{p}$ and $R^{*}=r^{*} I_{m}$, determined by

$$
\kappa^{*}=\sqrt{\frac{\left\|B^{T} B\right\|\left\|C^{T} C\right\|}{\left\|A^{T} A\right\|}\left(\frac{q^{*}}{r^{*}}\right)},
$$

provided the property of high-gain control still holds, that is, $P_{2} \approx P_{1}$, for

$\kappa_{2} / \kappa_{1}=\sqrt{\frac{\left\|B^{T} B\right\|\left\|C^{T} C\right\|}{\left\|A^{T} A\right\|}\left(\frac{q_{2}}{r_{2}}\right)} / \sqrt{\frac{\left\|B^{T} B\right\|\left\|C^{T} C\right\|}{\left\|A^{T} A\right\|}\left(\frac{q_{1}}{r_{1}}\right)}=\sqrt{\frac{q_{2}}{r_{2}}} / \sqrt{\frac{q_{1}}{r_{1}}}$ and $\kappa_{2}>\kappa_{1} \geq \kappa^{*}$, where $P_{2}$ and $P_{1}$ are the symmetric positive-definite solutions of the Riccati equations respectively.

$$
\begin{aligned}
A^{T} P_{1} & +P_{1} A+C^{T} Q_{1} C-\left(P_{1} B+C^{T} Q_{1} D\right)\left(R_{1}+D^{T} Q_{1} D\right)^{-1} \\
& \times\left(B^{T} P_{1}+D^{T} Q_{1} C\right)=0 \\
A^{T} P_{2} & +P_{2} A+C^{T} Q_{2} C-\left(P_{2} B+C^{T} Q_{2} D\right)\left(R_{2}+D^{T} Q_{2} D\right)^{-1} \\
& \times\left(B^{T} P_{2}+D^{T} Q_{2} C\right)=0
\end{aligned}
$$

Remark 2:

Due to the features of the system and the different types of references, if the given compression factor $\kappa\left(t^{*}\right)$ is not reasonable the modified control scheme might not effectively reduce the control input $u_{M}^{*}(t)$ to $u_{M}(t)$ to make it lie within the constraint bounds. The proposed condition describes the relationship between the compression boundary $\bar{\kappa}$ and the pair of weighting matrices $\{Q, R\}$ for the modified control system. In other words, designers can also apply such condition to determine a reasonable compression boundary $\bar{\kappa}$ by appropriately selecting the weighting matrices $\{Q, R\}$.

Another characteristic of the modified optimal control that indicates that the modified control input $u_{M}^{*}(t)$ approximates the conventional control input $u_{T}(t)$ is stated in the following. Consider the Riccati equation

$$
A^{T} P_{T}+P_{T} A-\left(P_{T} B+C^{T} Q D\right)\left(R+D^{T} Q D\right)^{-1}\left(B^{T} P_{T}+D^{T} Q C\right)+C^{T} Q C=0
$$

with respect to the conventional controller $u_{T}(t)$ which, based on assumption 1, equation (25) reduces to

$-\left(P_{T} B+C^{T} Q D\right)\left(R+D^{T} Q D\right)^{-1}\left(B^{T} P_{T}+D^{T} Q C\right)+C^{T} Q C \cong 0$.

Similarly, the Riccati equation corresponding to the modified controller $u_{M}^{*}(t)$ can be approximated as

$$
\begin{aligned}
& -\left(P_{M}\left(t^{*}\right)\left(\frac{B}{\kappa\left(t^{*}\right)}\right)+C^{T} Q\left(\frac{D}{\kappa\left(t^{*}\right)}\right)\right)\left(R+\left(\frac{D}{\kappa\left(t^{*}\right)}\right)^{T} Q\left(\frac{D}{\kappa\left(t^{*}\right)}\right)\right)^{-1} \\
& \times\left(\left(\frac{B}{\kappa\left(t^{*}\right)}\right)^{T} P_{M}\left(t^{*}\right)+\left(\frac{D}{\kappa\left(t^{*}\right)}\right)^{T} Q C\right)+C^{T} Q C \cong 0,
\end{aligned}
$$

i.e.

$$
-\left(P_{M}\left(t^{*}\right) B+C^{T} Q D\right)\left(\kappa^{T}\left(t^{*}\right) R \kappa\left(t^{*}\right)+D^{T} Q D\right)^{-1}\left(B^{T} P_{M}\left(t^{*}\right)+D^{T} Q C\right)+C^{T} Q C \cong 0 .
$$


From (26) and (27), the relationship between $P_{T}$ and $P_{M}\left(t^{*}\right)$ can be obtained as

$P_{M}\left(t^{*}\right) \approx P_{T} D^{T} Q D \gg \kappa^{T}\left(t^{*}\right) R \kappa\left(t^{*}\right)$

Then, according to (28), the feedback gain $K_{c M}\left(t^{*}\right)$ of the modified controller $u_{c M}^{*}(t)$ satisfies

$K_{c M}\left(t^{*}\right)=\left(\kappa\left(t^{*}\right) R \kappa\left(t^{*}\right)+D^{T} Q D\right)^{-1}\left(B^{T} P_{M}\left(t^{*}\right)+D^{T} Q C\right) \quad \cong\left(R+D^{T} Q D\right)^{-1}\left(B^{T} P_{T}+D^{T} Q C\right)=K_{c T}$,

if $D^{T} Q D \gg \kappa^{T}\left(t^{*}\right) R \kappa\left(t^{*}\right)$, i.e.

$K_{c M}\left(t^{*}\right) \approx K_{c T}$, if $D^{T} Q D \gg \kappa^{T}\left(t^{*}\right) R \kappa\left(t^{*}\right)$.

Furthermore, the forward gains $E_{c M}(t)$ and $E_{c T}(t)$ of these two controllers exhibit the property

$$
\begin{aligned}
E_{c M}\left(t^{*}\right)= & -\left(\kappa^{T}\left(t^{*}\right) R \kappa\left(t^{*}\right)+D^{T} Q D\right)^{-1}\left\{\left[\left(C-D K_{c M}\left(t^{*}\right)\right.\right.\right. \\
& \left.\left.\times\left(A-B K_{c M}\left(t^{*}\right)\right)^{-1} B-D\right]\right\}^{T} Q \\
\approx & -\left(R+D^{T} Q D\right)^{-1}\left\{\left[\left(C-D K_{c M}\left(t^{*}\right)\right)\right.\right. \\
& \left.\left.\times\left(A-B K_{c M}\left(t^{*}\right)\right)^{-1} B-D\right]\right\}^{T} Q \\
\approx & -\left(R+D^{T} Q D\right)^{-1} \times\left\{\left[\left(-D K_{c M}\left(t^{*}\right)\right)\left(-B K_{c M}\left(t^{*}\right)\right)^{-1} B-D\right]\right\} Q \cong E_{c T}
\end{aligned}
$$

if $D^{T} Q D \gg \kappa^{T}\left(t^{*}\right) R \kappa\left(t^{*}\right)$, i.e.

$$
E_{c M}\left(t^{*}\right) \cong E_{c T}, \text { if } D^{T} Q D \gg \kappa^{T}\left(t^{*}\right) R \kappa\left(t^{*}\right),
$$

where $\left\|B K_{c T}\right\| \gg\|A\|$ and $\left\|D K_{c T}\right\| \gg\|C\|$, which also implies

$$
E_{c T}=-\left(R+D^{T} Q D\right)^{-1}\left\{\left[\left(C-D K_{c T}\right)\left(A-B K_{c T}\right)^{-1} B-D\right]\right\}^{T} Q \approx-\left(R+D^{T} Q D\right)^{-1}\left\{\left[\left(-D K_{c M}\right)\left(-B K_{c M}\right)^{-1} B-D\right]\right\}^{T} Q .
$$

Hence, by combining (32) and (33) it can be deduced that

$$
u_{M}^{*}(t) \cong u_{T}(t) \text {, i.e. } u_{M}(t) \cong \frac{1}{\kappa\left(t^{*}\right)} u_{T}(t) .
$$

The actual input $u_{M}(t)$ can be effectively restricted to be within the pre-specified constraint interval by choosing a reasonable compression factor $\kappa\left(t^{*}\right)$, where the sufficient conditions for the case of a scalar SISO system are [16].

$$
\begin{aligned}
& \frac{q}{r} \gg \frac{a^{2}}{b^{2} c^{2}}, \\
& \frac{q}{r} \gg \frac{a}{(a d-b c) d}, \text { for } a d \neq b c, \\
& \left(\frac{q}{r}\right) \gg \frac{b^{2} c^{2}}{(a d-b c)^{2} d^{2}}, \text { for } a d \neq b c .
\end{aligned}
$$

Remark 3:

$$
\text { From (35b)-(35c), one has }
$$

$$
\left(\frac{q_{c}}{r_{c}}\right)^{2} /\left(\frac{q_{c}}{r_{c}}\right)=\left(\frac{a}{(a d-b c) d}\right)^{2} / \frac{b^{2} c^{2}}{(a d-b c)^{2} d^{2}},
$$

which implies that

$$
\frac{q_{c}}{r_{c}}=\frac{a^{2}}{b^{2} c^{2}} \text {. }
$$

Notice that (36) and (37) show that the upper bound $\bar{\kappa}$ is not related to the $d$ term, i.e. the $d$ term-free case. The upper bound $\bar{\kappa}$ of the compression factor $\kappa$ is then defined as

$$
\bar{\kappa}=\frac{1}{\alpha} \sqrt{\frac{b^{2} c^{2}}{a^{2}} \cdot \frac{q_{c}}{r_{c}}},
$$

under a positive constant $\alpha>1$.

Digital redesign of optimal control for sampled-data systems under input constraints:

If an analog controller is well designed, the corresponding digital controller can be easily determined, without altering the complete control scheme presented in Tsai et al. [10] using the prediction based-digital redesign technique. The whole modified prediction-based digital controller is depicted in Fig. 5.

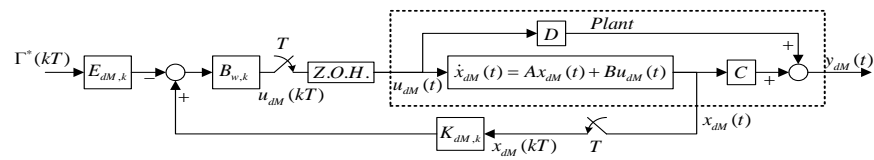




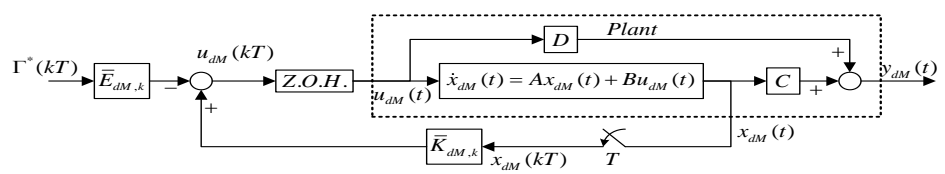

Fig. 5: Modified prediction-based digital controller, where (a) and (b) are equivalent.

The design procedure for the modified digital control scheme for proper systems with input constraints (which is omitted here) is similar to the design procedure for the strictly proper systems presented in Tsai et al. [10].

Lemma 2:

The anti-windup controller proposed above deals with the input constraints where $\bar{u}_{i}$ and $\underline{u}_{i}$ have opposite signs (i.e. $\bar{u}_{i} \times \underline{u_{i}}<0$ ). If $\bar{u}_{i}$ and $\underline{u}_{i}$ have the same sign (i.e. $\bar{u}_{i} \times \underline{u}_{i}>0$ ), the concept of shift operator can be applied to transform the problem into the standard one, i.e. to fit the proposed design procedure [10].

The design procedure for the FTC using the modified STC methodology with fault detection and covariance matrices resetting, and the modified digital control scheme for the case of input constraints is similar to the input-constraint-free case [21]. In order to limit the length of the paper, this procedure is omitted here. The structure of the proposed state-space self-tuning control with the (reduced-order) observer-based modified NARMAX model is shown in Fig. 6.

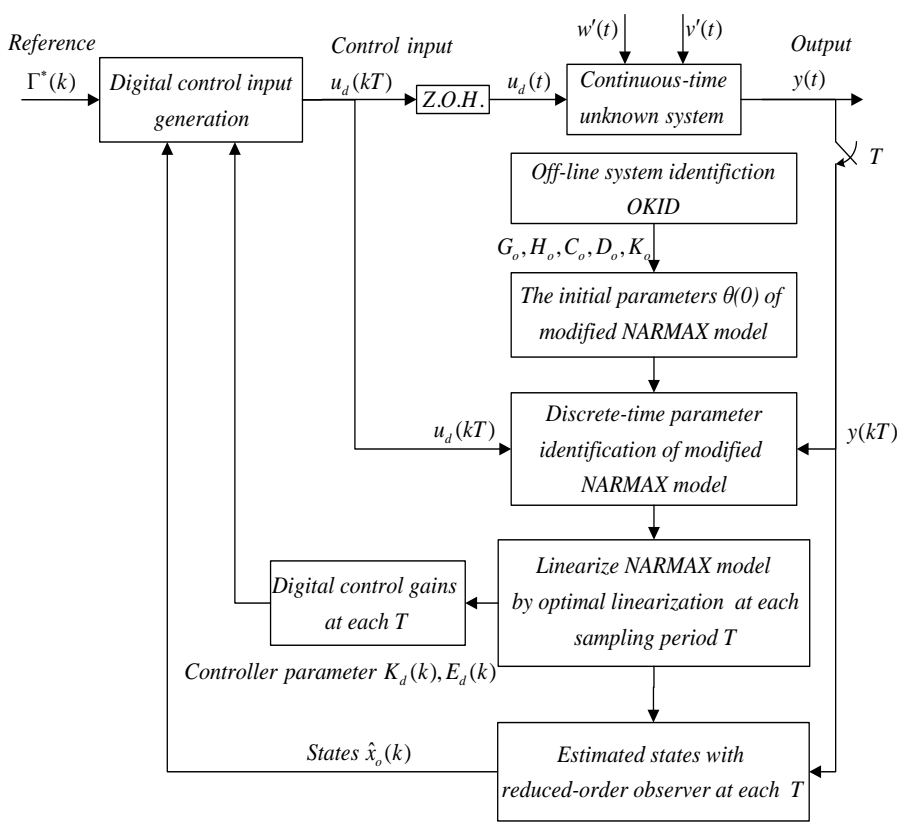

Fig. 6: Structure of the state-space self-tuning control with the (reduced-order) observer-based modified NARMAX model and OKID for unknown nonlinear stochastic system.

\section{An Illustrative Example:}

Control of a nonlinear system described by a NARMAX model:

Consider the large-scale interconnected sampled-data system (for $N=2$ ) with state-delay terms described by

System 1:

$$
\begin{aligned}
y_{11}(k)= & -0.4\left(0.2 y_{11}(k-1)+0.2 y_{12}(k-1) \sin \left(y_{11}(k-1)\right)\right. \\
& +y_{11}^{2}(k-2)+0.4 y_{11}(k-1) u_{11}(k-1)+u_{11}(k-1) \\
& \left.+0.8 e_{11}(k-1)+0.7 y_{11}(k-2) e_{11}(k-2)+u_{11}(k)\right) \\
& +e_{11}(k)+0.02 y_{21}(k-1)+0.01 y_{22}(k-1), \\
y_{12}(k)= & -0.3\left(0.2 y_{12}(k-1)+0.4 y_{12}(k-1) \cos \left(y_{12}(k-2)\right)\right. \\
& +y_{12}^{2}(k-2)+0.1 y_{12}(k-1) u_{12}(k-1)+u_{12}(k-1) \\
& +0.8 e_{12}(k-1)+0.5 y_{11}(k-2) e_{12}(k-2) \\
& \left.+u_{12}(k)\right)+e_{12}(k)+0.05 y_{21}(k-1), \\
\text { System } & 2: \\
y_{21}(k)= & -0.41\left(0.2 y_{21}(k-1)+0.2 y_{22}(k-1) \cos \left(y_{21}(k-1)\right)\right. \\
& +y_{21}^{2}(k-2)+0.4 y_{21}(k-1) u_{21}(k-1)+u_{21}(k-1) \\
& \left.+0.8 e_{21}(k-1)+0.7 y_{21}(k-2) e_{21}(k-2)+u_{21}(k)\right) \\
& +e_{21}(k)+0.1 y_{11}(k-1)+0.02 y_{12}(k-1), \\
y_{22}(k) & =-0.32\left(0.2 y_{22}(k-1)+0.3 y_{22}(k-1) \cos \left(y_{22}(k-2)\right)\right. \\
& +y_{22}^{2}(k-2)+0.1 y_{22}(k-1) u_{22}(k-1)+u_{22}(k-1) \\
& \left.+0.8 e_{22}(k-1)+0.5 y_{21}(k-2) e_{22}(k-2)+u_{22}(k)\right) \\
& +e_{22}(k)+0.05 y_{12}(k-1),
\end{aligned}
$$


Citation: Jason Sheng-Hong Tsai, Wen-Teng Hsu, Ya-Chiou Hsu, Shu-Mei Guo, Tzong-Jiy Tsai, Jose I. Canelon, Leang-San Shieh, 2018. An Input-Constrained NARMAX Model-Based Adaptive Tracker with Fault Tolerance for Unknown Systems with an Input-Output Direct Feed-Through Term. Advances in Natural and Applied Sciences. 12(3): 8-20.

where $y_{11}(k), y_{12}(k)$ and $y_{21}(k), y_{22}(k)$ are outputs, $u_{11}(k), u_{12}(k)$ and $u_{21}(k), u_{22}(k)$ are inputs, $e_{11}(k), e_{12}(k)$ and $e_{21}(k), e_{22}(k)$ are zero-mean Gaussian noise sequences with variances $\sigma_{e 11}^{2}=\sigma_{e 12}^{2}=\sigma_{e 21}^{2}=\sigma_{e 22}^{2}=0.01$. Given the NARMAX model in Appendix A, let system (39a)-(39b) be excited by control inputs $u_{1}(t)=\left[\begin{array}{ll}u_{11}(t) & u_{12}(t)\end{array}\right]^{T}$ and $u_{2}(t)=\left[\begin{array}{ll}u_{21}(t) & u_{22}(t)\end{array}\right]^{T}$, which are zero-mean white noise with covariance diag $\left(\operatorname{cov}\left(u_{i j}\right)=[0.10 .1]\right)$, where the sampling period $T_{S}$ is $0.01 \mathrm{sec}$. The system and observer gains $G_{o}, H_{o}, C_{O}, D_{o}$ and $K_{o}$ in Fig. 6 are indirectly obtained by the OKID method. The optimal linearization method is used to obtain initial parameters $\theta(0)$ closed to the convergence value of $\theta(k)$, where $\lambda_{0}=0.9, \lambda(0)=0.9$ and $S_{1}(0)=S_{2}(0)=I_{n_{\theta}}$. The weighting matrices are chosen as $\left\{Q=10^{8} \times I_{2}, R=I_{2}\right\}$ and $\left\{Q_{O}=10^{8} \times I_{4}, R_{O}=I_{2}\right\}$. The simulation results for the controlled decentralized system are shown in Fig. 7 .

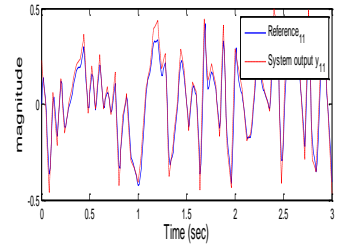

(a)

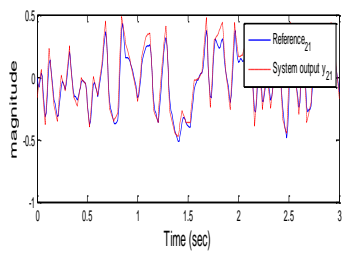

(b)
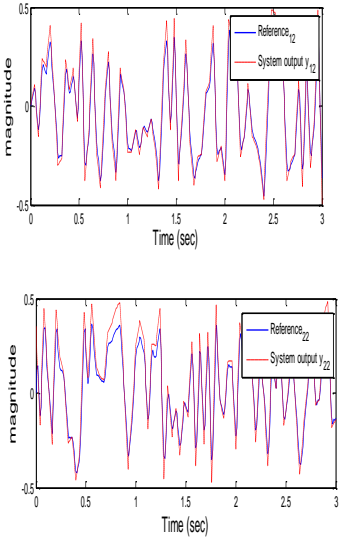

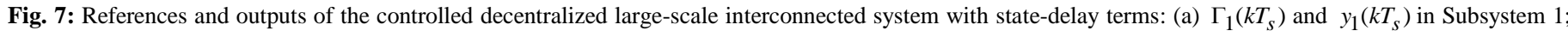
(b) $\Gamma_{2}\left(k T_{S}\right)$ and $y_{2}\left(k T_{s}\right)$ in Subsystem 2 .

For the case of no fault occurring, Figure 9 shows that the controlled outputs track the desired reference quickly and accurately with the high-gain decentralized observer-based digital-redesign trackers, for the unknown sampled-data interconnected large-scale system with state-delay terms.

Active fault tolerance using the modified NARMAX model-based state-space self-tuning control without input constraints:

The initial parameter $\theta(0)$ of the determined NARMAX model for the on-line RELS is obtained by off-line OKID. If a fault is detected at $t=k_{f}$, matrices $R_{1 i}, R_{2 i}$, and $\bar{S}_{i}\left(k_{f}\right)$ with the reset parameter $\delta=1$ in Tsai et al. [21] will be reset again automatically. Let the fault detection thresholds $\gamma_{\varepsilon 1}$ and $\gamma_{\varepsilon 2}$ be 3.0 [21]. The modified NARMAX model is used to identify the nonlinear system, and then activate the FTC at the first second. In the time interval from 1.5 seconds to the final time, Inputs 1 and 2 are suddenly increased 1.8 times in System 1, while System 2 has the same situation as System 1. The simulation results are shown below. Figure 8 corresponds to the situation without FTC and input constraint. Obviously, Subsystem 1 diverges immediately and Subsystem 2 has poor tracking performance.

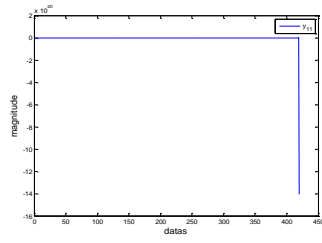

(a)

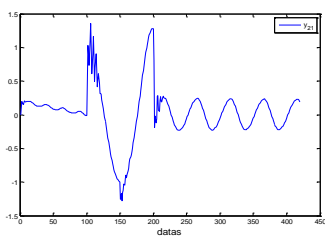

(b)

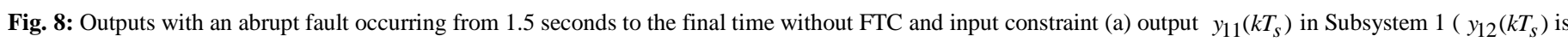
omitted at here); (b) output $y_{21}\left(k T_{s}\right)$ in Subsystem $2\left(\left(y_{22}\left(k T_{s}\right)\right.\right.$ is omitted at here).

With input constraints $u_{\text {sat }, i}^{I}=\left[\bar{u}_{i}, \underline{u}_{i}\right]=[2.45,-2.45]$ for $i=1$ and 2 , Figure 9 shows the simulation results for the case with FTC but without activating the proposed input-constraint mechanism, while Figure 10 involves both FTC and the proposed input-constraint mechanism.
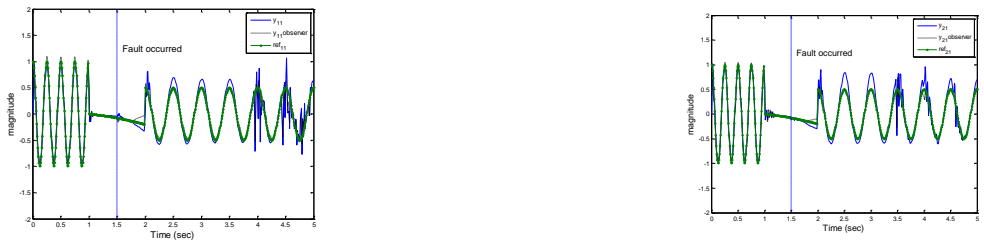

(a) 

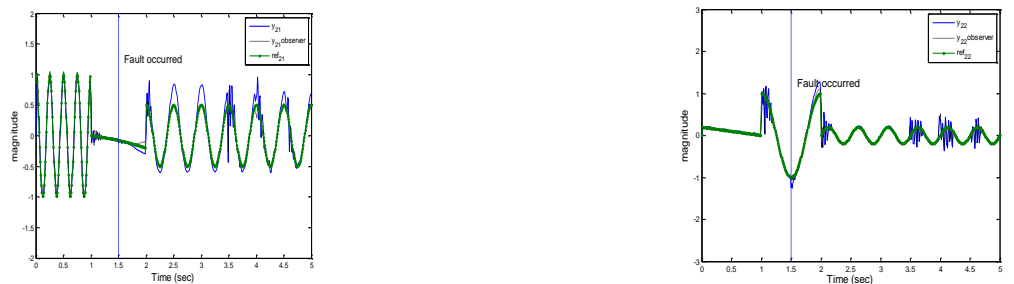

(b)

Fig. 9: Outputs for the case of an abrupt fault occurring in the time interval from 1.5 seconds to the final time, using FTC but without activating the proposed input-constraint mechanism: (a) reference $\Gamma_{1}\left(k T_{S}\right)$, observer output, and system output $y_{d 1}\left(k T_{S}\right)$ in Subsystem 1; (b) reference $\Gamma_{2}\left(k T_{S}\right)$, observer output, and system output $y_{d 2}\left(k T_{S}\right)$ in Subsystem 2 .

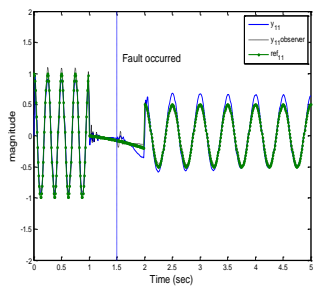

(a)

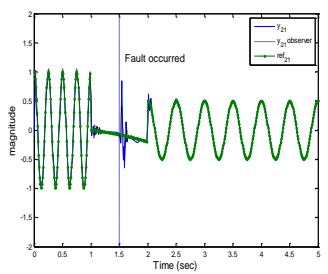

(b)
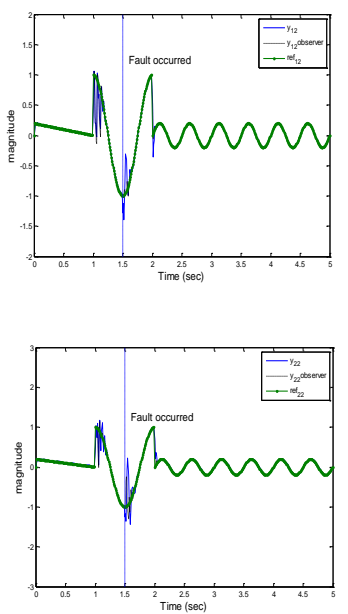

Fig. 10: Outputs for the case of an abrupt fault occurring in the time interval from 1.5 seconds to the final time, using FTC and activating the proposed inputconstraint mechanism: (a) reference $\Gamma_{1}\left(k T_{s}\right)$, observer output, and system output $y_{1}\left(k T_{S}\right)$ in Subsystem 1 ; (b) reference $\Gamma_{1}\left(k T_{S}\right)$, observer output, and system output $y_{2}\left(k T_{S}\right)$ in Subsystem 2 .

Simulation results indicate that the proposed control scheme could make the conventional control input comply with the corresponding constraints, for a specified reasonable compression factor (not shown to limit the paper length), and also show that it preserves the tracking performance of a well-designed conventional controller.

Conclusion:

This paper proposes a fault-tolerant state-space self-tuner with input constraints based on a modified NARMAX model in polynomial-expansion-form, for an unknown nonlinear stochastic hybrid systems with an input-output feed-through term. The main control schemes presented in this paper are i) An estimated timevarying system parameter and state-space model-based optimal tracker for an unknown nonlinear system with a direct transmission term, ii) An anti-windup scheme based on these models that sets up decentralized trajectory trackers for an unknown interconnected large-scale system with input constraints and state delays, iii) A control scheme that focuses on designing a high-performance active fault tolerant state-space self-tuner using the modified NARMAX model-based system identification, when the system has a fault.

\section{REFERENCES}

1. Warwick, K., 1981. Self-tuning regulators-A state-space approach. International Journal of Control, 33: 839-858.

2. Tsay, Y.T. and L.S. Shieh, 1981. State-space approach for self-tuning feedback control with pole assignment. IEE Proceeding D. Control Theory and Application, 123: 93-101.

3. Shieh, L.S., Y.L. Bao and F.R. Chang, 1989. State-space self-tuning regulators for general multivariable stochastic systems. IEE Proceeding D-Control Theory and Application, 136: 17-27.

4. Davies, R. and M.B. Zarrop, 1997. On the reduced variance over-parametrized pole-assignment self-tuning regulator. International Journal of Adaptive Control and Signal Processing, 11(8): 681-697.

5. Akhtar, S, and D.S. Bernstein, 2005. Lyapunov-stable discrete-time model reference adaptive control. International Journal of Adaptive Control and Signal Processing, 19(10): 745-767.

6. Wu, C.Y., J.S.H. Tsai, S.M. Guo, L.S. Shieh, J.I. Canelon, F. Ebrahimzadeh and L. Wang, 2015. A novel on-line observer/Kalman filter identification method and its application to input-constrained active fault-tolerant tracker design for unknown stochastic systems. Journal of the Franklin InstituteEngineering and Applied Mathematics, 352: 1119-1151.

7. Aström, K.J. and B. Wittenmark, 1989. Adaptive Control, NY, Addison-Wesley.

8. Leontaritis, I.J. and S.A. Billings, 1985. Input-output parametric models for nonlinear systems, Parts I and II. International Journal of Control, 41(2): 303344.

9. Li, J., W. Chen and Y. Fang, 2009. Adaptive NN output-feedback stabilization for a class of stochastic nonlinear strict-feedback systems. ISA Transactions, 48(4): 468-475.

11. Sontag, E.D., 1979. Polynomial Response Maps, 13, Berlin: Springer-Verlag.

10. Tsai, J.S.H., Y.Y. Du, S.M. Guo, C.W. Chen and L.S. Shieh, 2011. Optimal anti-windup digital redesign of MIMO control systems under input constraints. IET Control Theory and Applications, 5(3): 447-464.

12. Tsai, J.S.H., C.T. Wang, C.C. Kuang, S.M. Guo, L.S. Shieh and C.W. Chen, 2010. A NARMAX model-based state-space self-tuning control for nonlinear stochastic hybrid systems. Applied Mathematical Modeling, 34: 3030-3054. 
13. Wang, J.H., J.S.H. Tsai, J.S. Huang, S.M. Guo and L.S. Shieh, 2013. A low-order active fault-tolerant state space self-tuner for the unknown sampled-data nonlinear singular system using OKID and modified ARMAX model-based system identification. Applied Mathematical Modeling, 37 : $1242-1274$.

14. Tarbouriech, S. and M. Turner, 2009. Anti-windup design: an overview of some recent advances and open problems. IET Control Theory and Applications, 3(1): 1-19.

15. Valluri, S.and M. Soroush, 2003. A non-linear controller design method for processes with saturating actuators. International Journal Control, 76(7): 698716.

16. Zaccarian, L., D. Nesic and A.R. Teel, 2005. L2 anti-windup for linear dead-time systems. System Control Letter, 54(12): 1205-1217.

17. Mulder, E.F., P.Y. Tiwari and M.V. Kothare, 2009. Simultaneous linear and anti-windup controller synthesis using multiobjective convex optimization. Automatica, 45(3): 805-811.

18. Sawada, K., T. Kiyama and T. Iwasaki, 2009. Generalized sector synthesis of output feedback control with anti-windup structure. System Control Letter, 58(6): 421-428.

19. Kim, J.S., T.W. Yoon, H. Shim and J.H. Seo, 2008. Switching adaptive output feedback model predictive control for a class of input-constrained linear plants. IET Control Theory and Application, 2(7): 573-582.

20. Maeder, U., R. Cagienard and M. Morari, 2007. Explicit Model Predictive Control. Heidelberg: Springer-Berlin.

21. Tsai, J.S.H., W.T. Hsu, L.G. Lin, S.M. Guo and J.W. Tan, 2014. A modified NARMAX model-based self-tuner with fault tolerance for unknown nonlinear stochastic hybrid systems with an input-output direct feed-through term. ISA Transactions, 53: 56-75.

22. Tsai, J.S.H., C.Y. Wu, C.H. Lee, S.M. Guo and T.J. Su, 2016. A new optimal linear quadratic observer-based tracker under input constraint for the unknown system with a direct feed-through term. Optimal Control Application and Methods, 1: 34-71.

Appendix A: NARMAX Model:

The modified NARMAX model for an $m$-input $p$-output system is given by

$\tilde{y}=\left[\begin{array}{c}\tilde{y}_{1 \overline{0}} \\ \tilde{y}_{2 \overrightarrow{0}} \\ \vdots \\ \tilde{y}_{p \overline{0}}\end{array}\right]=\left[\begin{array}{c}F_{1}(Y)+B_{1}(Y) U+K_{\mathrm{e} 1}(Y) E \\ F_{2}(Y)+B_{2}(Y) U+K_{\mathrm{e} 2}(Y) E \\ \vdots \\ F_{p}(Y)+B_{p}(Y) U+K_{\mathrm{e} p}(Y) E\end{array}\right]$,

where

$Y=\left[\begin{array}{c}y(k-1) \\ y(k-2) \\ \vdots \\ y\left(k-n_{y}\right)\end{array}\right], \quad U=\left[\begin{array}{c}u(k) \\ u(k-1) \\ \vdots \\ u\left(k-n_{u}\right)\end{array}\right], \quad E=\left[\begin{array}{c}\varepsilon(k-1) \\ \varepsilon(k-2) \\ \vdots \\ \varepsilon\left(k-n_{u}\right)\end{array}\right]$,

$y_{i \vec{j}}=y_{i}(k-j), u_{i \vec{j}}=u_{i}(k-j), \varepsilon_{i \vec{j}}=\varepsilon_{i}(k-j)$,

$\mathrm{F}_{i}(Y)=a_{i, 1} \bar{f}_{1}(y)+a_{i, 2} \bar{f}_{2}(y)+\cdots+a_{i, l} \bar{f}_{l}(y)$,

$\bar{f}_{i}(y)$ are nonlinear polynomials for $i=1,2, \cdots, l$,

$$
\begin{aligned}
& B_{i}(Y)=\left[\begin{array}{lllll}
1 & y^{T}(k-1) & y^{T}(k-2) & \cdots & y^{T}\left(k-n_{y}\right)
\end{array}\right] \\
& \times\left[\begin{array}{cccc}
b_{i, 0,1} & b_{i, 0,2} & \cdots & b_{i, 0, m \times\left(n_{u}+1\right)} \\
b_{i, 1,1} & b_{i, 1,2} & \cdots & b_{i, 1, m \times\left(n_{u}+1\right)} \\
b_{i, 2,1} & b_{i, 2,2} & \cdots & b_{i, 2, m \times\left(n_{u}+1\right)} \\
\vdots & \vdots & \ddots & \vdots \\
b_{i, p \times n_{y}, 1} & b_{i, p \times n_{y}, 2} & \cdots & b_{i, p \times n_{y}, m \times\left(n_{u}+1\right)}
\end{array}\right], \\
& K_{e i}(Y)=\left[\begin{array}{lllll}
1 & y^{T}(k-1) & y^{T}(k-2) & \cdots & y^{T}\left(k-n_{y}\right)
\end{array}\right] \\
& \times\left[\begin{array}{cccc}
d_{i, 0,1} & d_{i, 0,1} & \cdots & d_{i, 0, m \times n_{e}} \\
d_{i, 1,1} & d_{i, 1,2} & \cdots & d_{i, 1, m \times n_{e}} \\
d_{i, 2,1} & d_{i, 2,2} & \cdots & d_{i, 2, m \times n_{e}} \\
\vdots & \vdots & \ddots & \vdots \\
d_{i, p \times n_{y}, 1} & d_{i, p \times n_{y}, 2} & \cdots & d_{i, p \times n_{y}, m \times n_{e}}
\end{array}\right], \\
& \tilde{y}_{i 0}=a_{i, 1} \bar{f}_{1}(y)+a_{i, 2} \bar{f}_{2}(y)+\cdots \\
& +a_{i, l} \bar{f}_{l}(y)+b_{i, 0,1} u_{1 \overrightarrow{0}}+b_{i, 1,1} u_{1 \overrightarrow{0}}+b_{i, 2,1} u_{1 \overrightarrow{0}}+\cdots \\
& +d_{i, 0,1} \varepsilon_{1 \overrightarrow{1}}+d_{i, 1,1} y_{1 \overrightarrow{1}} \varepsilon_{1 \overrightarrow{1}}+\cdots
\end{aligned}
$$


$=\theta_{i}^{T} \phi_{i}(k)=\left[\begin{array}{c}a_{i, 1} \\ a_{i, 2} \\ \vdots \\ a_{i, l} \\ \hdashline b_{i, 0,1} \\ b_{i, 1,1} \\ \vdots \\ b_{i, p \times n_{y}, m \times n_{u}} \\ \hdashline d_{i, 0,1} \\ d_{i, 1,1} \\ \vdots \\ d_{i, p \times n_{y}, m \times n_{e}}\end{array}\right]^{T}\left[\begin{array}{c}\bar{f}_{1}(y) \\ \bar{f}_{2}(y) \\ \vdots \\ \bar{f}_{l}(y) \\ \hline u_{1 \overline{0}} \\ y_{1 \overline{1}} u_{1 \overline{0}} \\ \vdots \\ y_{p, \bar{n}_{y}} u_{m, \bar{n}_{u}} \\ \hdashline \varepsilon_{1 \overline{1}} \\ y_{1 \overline{1}} \varepsilon_{1 \overline{1}} \\ \vdots \\ y_{p, \bar{n}_{y}} \varepsilon_{m, \bar{n}_{e}}\end{array}\right]$

$=\left[\begin{array}{c}\theta_{A R M A X} \\ \theta_{\text {NAARMAX-nonlinear }}\end{array}\right]^{T}\left[\begin{array}{c}\phi_{A R M A X} \\ \phi_{\text {NAARMAX -nonlinear }}\end{array}\right]$,

$\phi_{\text {ARMAX }}=\left[\begin{array}{c}y(k-1)_{p \times 1} \\ y(k-2)_{p \times 1} \\ \vdots \\ y\left(k-n_{y}\right)_{p \times 1} \\ u(k)_{m \times 1} \\ u(k-1)_{m \times 1} \\ \vdots \\ u\left(k-n_{u}\right)_{m \times 1} \\ \varepsilon(k-1)_{p \times 1} \\ \varepsilon(k-2)_{p \times 1} \\ \vdots \\ \varepsilon\left(k-n_{e}\right)_{p \times 1}\end{array}\right]=\left[\begin{array}{c}Y_{\text {ARMAX }} \\ U_{\text {ARMAX }} \\ E_{\text {ARMAX }}\end{array}\right]$.

Let

$\bar{\phi}_{\text {NARMAX -nolinear }}=\phi_{A R M A X} Y_{A R M A X}^{T}$

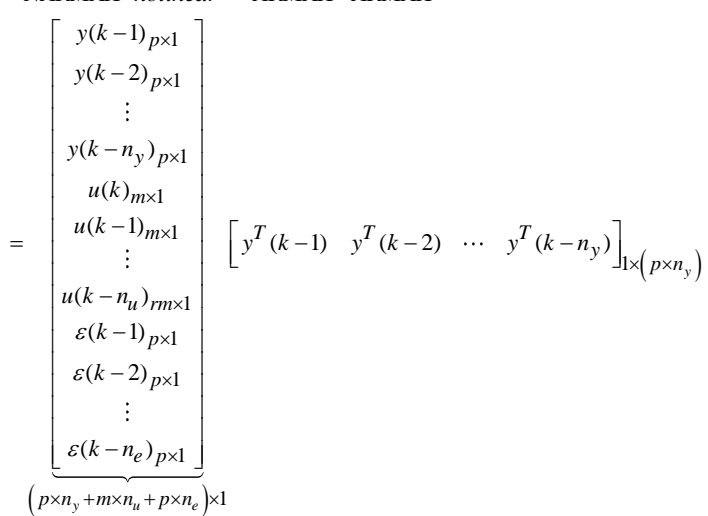

$=\left[\begin{array}{cccc}y(k-1) y(k-1) & y(k-1) y(k-2) & \cdots & y(k-1) y\left(k-n_{y}\right) \\ y(k-2) y(k-1) & y(k-2) y(k-2) & \cdots & y(k-2) y\left(k-n_{y}\right) \\ \vdots & \vdots & \ddots & \vdots \\ y\left(k-n_{y}\right) y(k-1) & y\left(k-n_{y}\right) y(k-2) & \cdots & y\left(k-n_{y}\right) y\left(k-n_{y}\right) \\ \hline u(k) y(k-1) & u(k) y(k-2) & \cdots & u(k) y\left(k-n_{y}\right) \\ u(k-1) y(k-1) & u(k-1) y(k-2) & \cdots & u(k-1) y\left(k-n_{y}\right) \\ \vdots & \vdots & \ddots & \vdots \\ u\left(k-n_{u}\right) y(k-1) & u\left(k-n_{u}\right) y(k-2) & \cdots & u\left(k-n_{u}\right) y\left(k-n_{y}\right) \\ \varepsilon(k-1) y(k-1) & \varepsilon(k-1) y(k-2) & \cdots & \varepsilon(k-1) y\left(k-n_{y}\right) \\ \varepsilon(k-2) y(k-1) & \varepsilon(k-2) y(k-2) & \cdots & \varepsilon(k-2) y\left(k-n_{y}\right) \\ \vdots & \vdots & \ddots & \vdots \\ \varepsilon\left(k-n_{e}\right) y(k-1) & \varepsilon\left(k-n_{e}\right) y(k-2) & \cdots & \varepsilon\left(k-n_{e}\right) y\left(k-n_{y}\right)\end{array}\right] \triangleq[(i, j)]_{\left(p \times n_{y}+m \times n_{u}+p \times n_{e}\right) \times\left(p \times n_{y}\right)}$.

Then, rearrange the $\bar{\phi}_{N A R M A X}$-nolinear from matrix form to vector form given as 
Citation: Jason Sheng-Hong Tsai, Wen-Teng Hsu, Ya-Chiou Hsu, Shu-Mei Guo, Tzong-Jiy Tsai, Jose I. Canelon, Leang-San Shieh, 2018. An Input-Constrained NARMAX Model-Based Adaptive Tracker with Fault Tolerance for Unknown Systems with an Input-Output Direct Feed-Through Term. Advances in Natural and Applied Sciences. 12(3): 8-20.

\begin{tabular}{|c|c|}
\hline \multirow[t]{2}{*}{$\phi_{N A R M A X-\text {-nolinear }}=$} & {$\left[\begin{array}{c}(1,1) \\
(1,2) \\
\vdots \\
\left(1, p \times n_{y}\right) \\
(2,1) \\
(2,2) \\
\vdots \\
\left(2, p \times n_{y}\right) \\
\vdots \\
\left(p \times n_{y}+m \times n_{u}+p \times n_{e}, 1\right) \\
\left(p \times n_{y}+m \times n_{u}+p \times n_{e}, 2\right) \\
\vdots \\
\left(p \times n_{y}+m \times n_{u}+p \times n_{\rho}, p \times n_{y}\right)\end{array}\right]$} \\
\hline & $p \times n_{y}\left(p \times n_{y}+m \times n_{u}+p \times n_{e}\right) \times 1$ \\
\hline
\end{tabular}

